\title{
Screening of Methicillin Resistant Staphylococcus aureus (MRSA) from Wounds in Pediatric Patients Visiting Tertiary Care in Hospital
}

\author{
Ganesh Bhandari ${ }^{1}$, Basant Pokhrel $^{1}$, Yogesh Oli², Archana Katuwal², Netra Lal Bhandari ${ }^{\star}$
}

${ }^{1}$ Department of Chemistry, Tri-Chandra Multiple Campus, Tribhuvan University, Kathmandu, Nepal ${ }^{2}$ Department of Microbiology, Tri-Chandra Multiple Campus, Tribhuvan University, Kathmandu, Nepal

\begin{abstract}
The extent of methicillin-resistant Staphylococcus aureus (MRSA) in children is still unknown. The collected wound pus samples were processed. Identification of $S$. aureus was done according to standard microbiological procedures as per the clinical laboratory standards institute (CLSI) guidelines (2016). The antibiogram of the isolates was carried out by the Kirby-Bauer disc diffusion technique. MRSA was determined by measuring the zone of inhibition (ZOI) surrounding to cefoxitin disc, with resistance defined as ZOI of $\leq 21 \mathrm{~mm}$. Out of 357 bacterial culture-positive samples, 278 (77.87 \%) were S. aureus isolates, among them $102(36.69 \%)$ were found to be MRSA. The percentage of MRSA isolates was found high in male children and inpatients with $61.76 \%$ and $73.52 \%$ respectively. All the MRSA isolates were susceptible to gentamicin $(79.41 \%)$, whereas $(91.17 \%)$ were resistant to penicillin. The distribution of MRSA in inpatients $75(73.52 \%)$ is higher than that of outpatients $27(26.74 \%)$. This study shows that the MRSA occurrence is prevalent in pediatric patients.
\end{abstract}

Keywords: Staphylococcus aureus, MRSA, Antibiotic susceptibility test, Disc-diffusion, Methicillin resistant

*Corresponding author

Email: netra.tu.edu@gmail.com

\section{Introduction}

Staphylococcus aureus (S. aureus) is one of the important human pathogen known over the past several decades. It is the cause of hospital and community-acquired infections [1]. It is one of the versatile nosocomial pathogens worldwide causing skin infections to life-threatening systemic illnesses including pneumonia, osteomyelitis and endocarditis [2,3]. S. aureus is one of the common and important Gram-positive hospital-acquired organisms. It can cause infection; most commonly at sites of lowered host resistance such as damaged skin or mucosal membranes, infections like pimples, boils and abscesses to severe systemic infections like bacteremia, endocarditis, pneumonia and toxic shock syndrome $[4,5]$.

S. aureus resistant to oxacillin, methicillin and a few others related antibiotics are all known under the generic term methicillin-resistant $S$. aureus. The penicillin-resistance $S$. aureus (PRSA) is typically coincides with the emergence of MRSA [6]. MRSA is a global public health problem, associated with considerable morbidity and mortality, causing both hospital and communityacquired infections [7]. Among the many species of antibiotic-resistant bacteria, MRSA is one of the most important causes of antibiotic treatment failure, increased morbidity and mortality. $S$. aureus has a remarkable ability to colonize the skin and mucous membranes. Hospitalized patients and health-care workers have higher colonization rates than the general population [8]. Studies revealed that nearly $6 \%$ hospital personnel and nearly $3 \%$ outpatients carry MRSA [9-12].

The prevalence of MRSA has varied from hospital to hospital in various countries. Several types of research and studies conducted in Nepal about $S$. aureus and their antibiotic susceptibility pattern suggest the gradual emergence of MRSA in hospitals. However, only a few reports related to MRSA are available in Nepal which is carried out in different hospitals at different periods. In a bacteriological study carried out at Tribhuvan University Teaching Hospital (TUTH), the prevalence of MRSA was found to be $23.5 \%$ [13]. A similar study carried out at Kanti Children's 
Hospital showed $31.4 \%$ of MRSA isolates and $11.7 \%$ from TUTH [14]. Rajbhandari et al. (2002) reported 54.9\% strains of MRSA [15]. Similarly, Kumari et al. (2008) reported 26.14\% MRSA strains in a study carried out in a tertiary- care hospital in Eastern Nepal [16]. Sanjana et al. (2010) also reported 39.6\% Methicillin-resistant $S$. aureus isolates at the College of Medical SciencesTeaching Hospital, Chitwan. Tiwari et al. (2009) reported MRSA isolates were resistant to cotrimoxazole was $77 \%$ and $81.7 \%$ (Ansari et al. 2014). Pant et al. (2018) reported $82.6 \%$ MRSA resistance to chloramphenicol [17-20].

The prime focus of the study is the distribution and prevalence of $S$. aureus and MRSA in wound samples collected in the microbiology laboratory of International Friendship Children's Hospital as well as on its antibiotic sensitivity pattern. The study will also demonstrate the present scenario of MRSA in children and the sensitivity pattern of different antibiotics used against it. This is useful for future planning and policy making in health care centers and hospitals in order to combat the spreading of infectious diseases.

\section{Materials and Methods Sample collection and transportation}

Clinical specimens were collected, stored, transported and processed in the laboratory immediately after collection by following standard microbiology guidelines [21]. The sterile swab was moved across the surface of wounds in a zigzag motion and pus aspirates were collected aseptically by puncturing the wound using a sterile syringe. The accurate, rapid microbiological diagnosis of MRSA and $S$. aureus infections begins with proper specimen collection and rapid transportation to the laboratory. To make sure the collection of the best possible specimen, the Health Care Workers (HCWs) must be properly trained, and the patient must be provided with clearly presented and fully understood instruction for sample collection.

\section{Specimen processing}

Pus samples received in the microbiology laboratory were processed as per routine standard microbiological procedures described by the established guideline for the isolation and identification of S. aureus [22].

\section{Culture of specimen}

All the pus samples were inoculated on blood agar (BA) and Mac-conkey agar (MA) plates and incubated aerobically at $37^{\circ} \mathrm{C}$ for 24 to 48 hours in the incubator. If no growth was observed, it was reported as growth negative. The suspected $S$. aureus isolates (on the basis of haemolysis produced on BA) were inoculated in to mannitol salt agar (MSA) to check if the isolates ferment mannitol or not. Photograph of $S$. aureus culture on blood agar and MSA is pictured in figure 1.
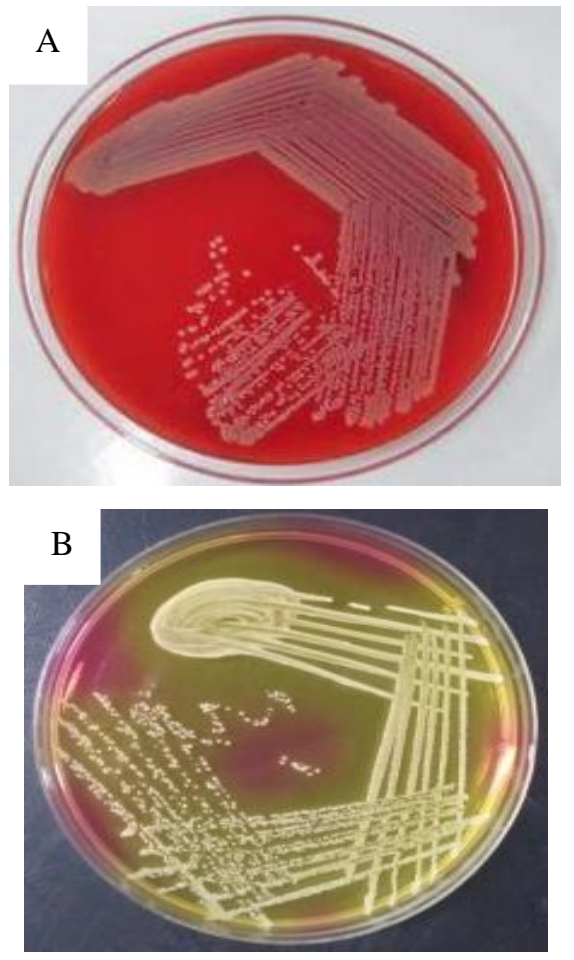

Figure 1. Photograph of S. aureus culture on (A) blood agar (B) mannitol salt agar (MSA)

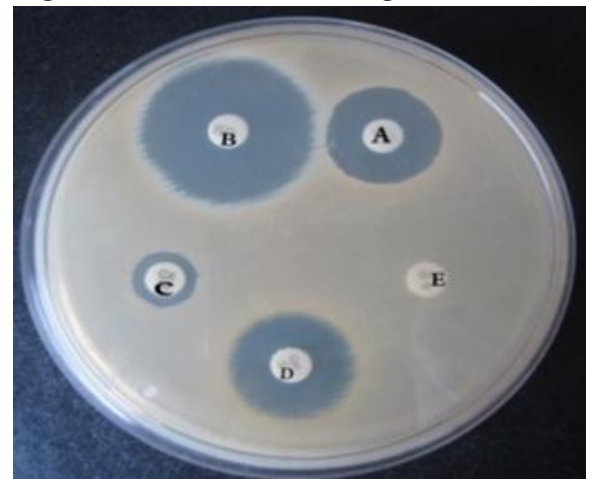

Figure 2. Photograph of antibiotic sensitivity test of S. aureus on MHA. A: Gentamicin (30 mcg), B: Chloramphenicol (30mcg), C: Cefoxitin (30mcg), D: Ciprofloxacin $(5 \mathrm{mcg})$, E: Penicillin $(10 \mathrm{mcg})$ 


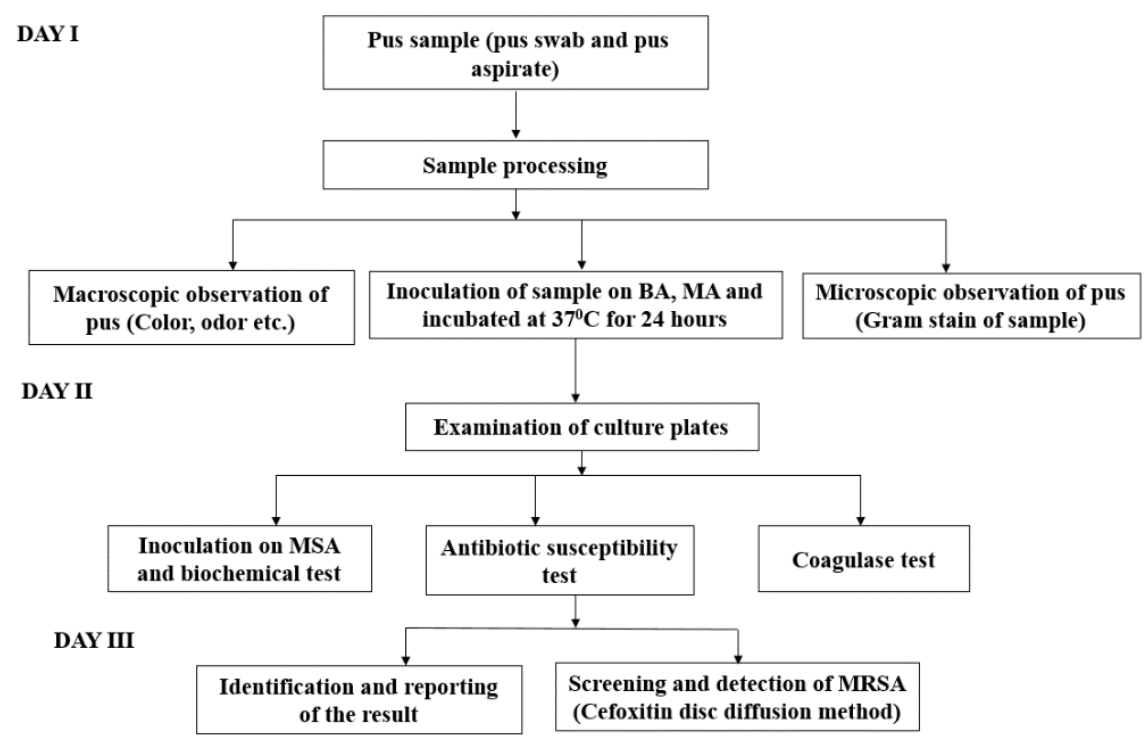

Figure 3. Flow chart representing isolation and identification of MRSA from pus samples

\section{Identification of the isolates}

The growth was identified as $S$. aureus by using conventional biochemical methods as per standard microbiological techniques described in Bergey's Manual of Determinative Bacteriology [23]. It comprises colonial morphology, staining reactions and various biochemical properties such as catalase-positive, coxidase negative, fermentative, Voges-Proskauer positive, etc.

\section{Preservation of isolates}

Isolates in pure culture were preserved in glycerol $20 \%(\mathrm{v} / \mathrm{v})$ in brain heart infusion broth (BHI) at $-20{ }^{\circ} \mathrm{C}$ to $-70^{\circ} \mathrm{C}$ and recovered by sub culturing in brain heart infusion broth at $37^{\circ} \mathrm{C}$ for $24 \mathrm{hrs}$ followed by further subculture on nutrient agar.

\section{Antimicrobial susceptibility testing of S. aureus}

Antibiotic susceptibility testing of isolates was assessed by the modified Kirby-Bauer disk diffusion method as recommended by the Clinical and Laboratory Standard Institute using Mueller Hinton Agar (MHA) [22]. Using a sterile wire loop, a single isolated colony of which the sensitivity pattern is to be determined was touched and inoculated into a nutrient broth (NB) tube and was incubated for 2-4 hours. After incubation in a good light source, the turbidity of the suspension was matched with the turbidity of the standard of Mac Farland 0.5 (Prepared by adding $0.6 \mathrm{~mL}$ of $1 \% \mathrm{w} / \mathrm{v}$ barium chloride solution to $99.4 \mathrm{~mL}$ of $1 \% \mathrm{v} / \mathrm{v}$ solution of sulphuric acid) [24]. Using a sterile swab, a plate of Mueller Hinton agar was inoculated with bacterial suspension using carpet culture technique and plates were taken for incubation at $37^{\circ} \mathrm{C}$ for $18-24$ hours. The result was interpreted as whether the organism was sensitive or resistant to the tested antimicrobial agents. Photograph of antibiotic sensitivity test of $S$. aureus on MHA is shown in figure 2.

\section{Detection of MRSA}

MRSA detection was done by the cefoxitin disc $(30 \mu g)$ diffusion test. As per CLSI (2015) guideline, a lawn culture was made on Mueller Hinton Agar (MHA) supplemented with 4\% $\mathrm{NaCl}$ and cefoxitin disc from the suspension of turbidity equivalent to 0.5 Mc Farland standards from overnight growth in nutrient agar and incubated for 24 hours aerobically at $35^{\circ} \mathrm{C}$. After incubation, the plates were examined for zone of inhibition. Isolates exhibiting zone of inhibition $\geq$ $22 \mathrm{~mm}$ were considered as sensitive, whereas those showing zone of inhibition $\leq 21 \mathrm{~mm}$ were considered as resistant and were reported as MRSA.

\section{Purity plate}

The purity was used to ensure that the inoculation used for the biochemical tests was 
Table 1. Bacterial growth in different pus samples

\begin{tabular}{lccc}
\hline $\begin{array}{l}\text { Types of } \\
\text { sample }\end{array}$ & $\begin{array}{c}\text { Growth } \\
\text { (No. \%) }\end{array}$ & $\begin{array}{c}\text { No growth } \\
\text { (No. \%) }\end{array}$ & Total \\
\hline $\begin{array}{l}\text { Pus } \\
\text { swab }\end{array}$ & $303(55.09 \%)$ & $247(44.91 \%)$ & 550 \\
$\begin{array}{l}\text { Pus } \\
\text { aspirate }\end{array}$ & $54(54 \%)$ & $46(46 \%)$ & 100 \\
Total & $357(54.92)$ & $293(45.08)$ & $650(100)$ \\
\hline
\end{tabular}

Table 2. Gender wise distribution of patients $(\mathrm{P}>0.05)$

\begin{tabular}{lccc}
\hline & Male & Female & Total \\
\hline $\begin{array}{l}\text { Culture } \\
\text { positive }\end{array}$ & $196(54.9)$ & $161(45.09)$ & $357(54.9)$ \\
$\begin{array}{l}\text { Culture } \\
\text { negative }\end{array}$ & $162(45.2)$ & $131(44.8)$ & $293(45.1)$ \\
\hline Total & $358(55.1)$ & $292(44.9)$ & $650(100)$ \\
\hline
\end{tabular}

pure culture and also to see whether the biochemical tests were performed in aseptic condition or not. Thus, while performing biochemical tests, in biochemical media, the same inoculum was sub-cultured on one half of Nutrient Agar (NA) medium before (pre purity) inoculation and another half after (post purity) inoculation. The maintenance of aseptic condition is indicated by the growth of the same organism in pure form in both pre and post halves of the medium. The Chart below represents the isolation and identification of MRSA from pus samples.

All the raw data collected in the microbiology laboratory were statistically analyzed by using computer-based software program SPSS version 20. The flow chart representing isolation and identification of MRSA from pus samples is shown in figure 3.

\section{Results and Discussion}

Bacterial growth in different pus samples and gender wise distribution of patients

Out of 650 total samples, 550 samples were pus swab and 100 samples were pus aspirate. Among total pus swabs $303(55.09 \%)$ were culture positive and 247 (44.91\%) showed no growth. Moreover, among total pus aspirates, 54 (54\%) were culture positive and the remaining 46 (46\%) showed no growth as shown in Table 1.

Out of total cases, $358(55.07 \%)$ were male patients and $292(44.92 \%)$ were female patients. The growth was found to be higher in male patients $196(54.9 \%)$ than in female patients 161 (45.09\%). The association between gender and growth of bacteria was not statistically significant ( $p$-value $>0.05$ ) is presented in Table 2.

Among 650 pus samples, the majority i.e. 352 $(54.15 \%)$ were In-patients who were admitted to the different departments in the hospital and 298 $(45.23 \%)$ were from OPD. A total $221(62.78 \%)$ of In- Patient Department (IPD) and 136 (45.36 \%) of Out Patient Department (OPD) showed growth. Such distribution pattern of growth on the basis of the department was statistically significant ( $p$ value $<0.05$ ) is as in table 3 .

\section{Distribution of $S$, aureus in pus sample}

Among the culture-positive specimens, 278 (77.87\%) S. aureus isolates were obtained while remaining $79(22.12 \%)$ were other bacterial isolates which include both gram-positive Coagulase Negative Staphylococcus species (CoNS) and gram-negative bacteria (E. coli, Pseudomonas aeruginosa, Klebsiella pneumonia and Enterobacter aerogens) as shown in pie chart in figure 4.

\section{Distribution of clinical isolates of $S$. aureus according to age and gender of the patient}

Among 278 S. aureus isolates, a higher number of isolation was found in male $169(60.79 \%)$ as compared to female $109(39.20 \%)$ patients. In the male, the maximum number of $S$. aureus isolates $(\mathrm{n}=87,51.47 \%)$ was observed in the patient with an age group of 1-5 and in females, the maximum

Table 3. Distribution of bacterial growth on the basis of hospital Department

\begin{tabular}{lcccc}
\hline Organism & IPD $\{\mathrm{n}(\%)\}$ & OPD $\{\mathrm{n}(\%)\}$ & Total $\{\mathrm{n}(\%)\}$ & p-value \\
\hline $\begin{array}{l}\text { Culture } \\
\text { positive }\end{array}$ & $221(62.78)$ & $136(45.36)$ & $357(54.92)$ & \\
$\begin{array}{l}\text { Culture } \\
\text { negative }\end{array}$ & $131(37.21)$ & $162(54.36)$ & $293(45.07)$ & $\mathrm{P}<0.05$ \\
\hline Total & $352(54.15)$ & $298(45.84)$ & $650(100)$ & \\
\hline
\end{tabular}




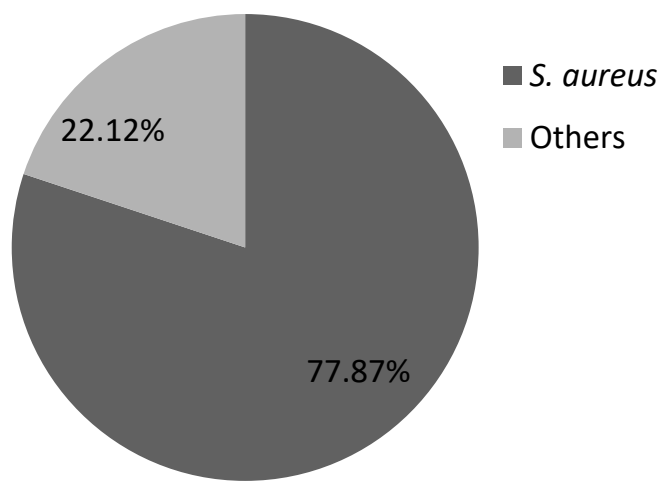

Figure 4. Distribution of $S$. aureus in pus sample. number of $S$. aureus isolates $(n=55,50.45 \%)$ was observed in the same 1-5 age group patients. Likewise, the least number of $S$. aureus isolates $(n=12,7.10 \%)$ was observed in $11-15$ age group patient in male and female least number of $S$. aureus isolates $(\mathrm{n}=9,8.25 \%)$ was observed in the same 11-15 age group patients is shown in Table 4.

Table 4. Distribution of clinical isolates of $S$. aureus according to age and gender of the patient

\begin{tabular}{lcccc}
\hline \multirow{2}{*}{$\begin{array}{l}\text { Age } \\
\text { group(in } \\
\text { years) }\end{array}$} & Male & Female & No. & $\%$ \\
\cline { 2 - 5 } Below 1 & 32 & 18 & 50 & 17.98 \\
$1-5$ & 87 & 55 & 142 & 51.07 \\
$6-10$ & 38 & 27 & 65 & 23.38 \\
$11-15$ & 12 & 9 & 21 & 7.55 \\
Total & $169(60.79 \%)$ & $109(39.2 \%)$ & 278 & 100.0 \\
\hline
\end{tabular}

\section{Comparison of clinical isolates of $S$.} aureus in inpatients and outpatients

Among 278 S. aureus isolates, 177 (63.66 \%) isolates of $S$. aureus were from inpatients whereas $101(36.33 \%)$ S. aureus were from outpatients. Similarly, among 79 growths other than $S$. aureus isolates, $47(59.49 \%)$ and $32(40.50 \%)$ cases of cultures other than $S$. aureus were found from inpatients and outpatients respectively. A comparison of clinical isolates of $S$. aureus in inpatients and outpatients is pictured in figure 5.

Distribution of MRSA among $S$. aureus and in different age groups

Out of 357 culture-positive isolates, 278 (77.87 \%) were S. aureus. Out of total S. aureus; 102 (36.69\%)

Table 5. Distribution of MRSA in outpatients and inpatients

\begin{tabular}{lcccc}
\hline & Inpatients (No. \%) & Outpatients (No. \%) & Total (No. \%) & P-value \\
\hline MRSA & $75(73.52 \%)$ & $27(26.47 \%)$ & $102(36.69 \%)$ & \\
MSSA & $101(57.38 \%)$ & $75(42.61 \%)$ & $176(63.30 \%)$ & $\mathrm{P}<0.05$ \\
\hline Total & 176 & 102 & $278(100.0)$ & \\
\hline
\end{tabular}

were MRSA and 176 (63.30 \%) were MSSA (Methicillin-sensitive Staphylococcus aureus). Out of $169(60.79 \%)$ S. aureus isolates among male patients, $63(61.76 \%)$ were found to be MRSA. Likewise, among 109 (39.20\%) S. aureus isolates among female patients, 39 (38.23\%) were found to be MRSA. Such distribution of MRSA based on gender was not statistically significant ( $p$-value; $>0.05)$ is given in the pie chart in figure 6 .

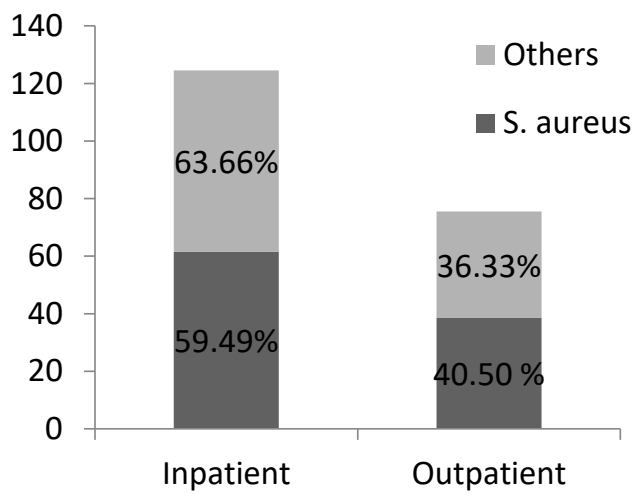

Figure 5. Comparison of clinical isolates of $S$. aureus in inpatients and outpatients

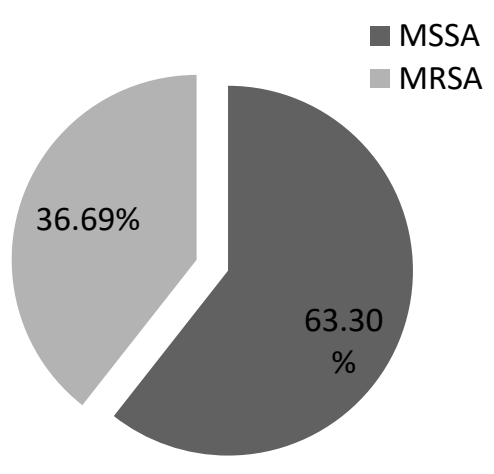

Figure 6. Distribution of MRSA among $S$. aureus

Out of 278 S. aureus isolates, altogether 102 $(36.69 \%)$ and $176(63.30 \%)$ were identified as MRSA and MSSA respectively by cefoxitin disc diffusion test which constituted 75 (73.52\%) MRSA and 101 (57.38\%) MSSA in admitted patients. Similarly, in outpatients, 27(26.47\%) MRSA and 75(42.61\%) MSSA were observed. The association in the MRSA occurrence and inpatients was statistically significant $(p=0.024)$ is in Table 5 
Table 6. Antibiotic susceptibility pattern of S. aureus isolates

\begin{tabular}{lcccc}
\hline \multirow{2}{*}{$\begin{array}{l}\text { Untibiotics } \\
\text { Used }\end{array}$} & \multicolumn{4}{c}{ Total No of S. aureus isolates $=278$} \\
\cline { 2 - 5 } & Sensitive & $\%$ & Resistant & $\%$ \\
\hline Amikacin & 236 & 84.89 & 42 & 15.10 \\
Ciprofloxacin & 100 & 35.97 & 178 & 64.02 \\
Cotrimoxazole & 125 & 44.96 & 153 & 55.04 \\
Cefoxitin & 176 & 63.30 & 102 & 36.69 \\
Erythromycin & 166 & 59.7 & 112 & 40.28 \\
Cloxacillin & 180 & 64.74 & 98 & 35.25 \\
Cefotaxime & 236 & 84.89 & 42 & 15.10 \\
Penicillin & 28 & 10.08 & 250 & 89.92 \\
Ofloxacin & 116 & 41.72 & 162 & 58.27 \\
Chloramphenicol & 255 & 91.72 & 23 & 8.27 \\
Gentamicin & 264 & 94.96 & 14 & 5.03
\end{tabular}

Antibiotic susceptibility pattern of $S$. aureus isolates

The antibiotic susceptibility pattern showed that the highest number of isolates were resistant to penicillin $(n=250,89.92 \%)$, ciprofloxacin $(n=178$, $64.02 \%)$ ofloxacin $(\mathrm{n}=162, \quad 58.27 \%)$, cotrimoxazole $(\mathrm{n}=153,55.04 \%)$, erythromycin $(n=112,40.28 \%)$, cefoxitin $(n=102,36.69 \%)$ and cloxacillin ( $n=98,35.25 \%)$. The highest number of penicillin resistant $S$. aureus developed the ability to inactivate the $\beta$ - lactam ring of penicillin by producing plasmid encoded $\beta$ - lactamase [25]. Similarly, highest number of MRSA isolates were susceptible to gentamicin $(n=264,94.96 \%)$ is due to the combination $\beta$ - lactam and amino glycosides which increases bacterial killing invitro and in animal model of endocarditis, followed by chloramphenicol ( $\mathrm{n}=255,91.72 \%)$, cefotaxime $(n=236,84.89 \%)$ and amikacin $(n=236$, $84.89 \%)$, cloxacillin $(\mathrm{n}=180,64.74 \%)$, cefoxitin $(n=176,63.30 \%)$, erythromycin $(n=166$, $59.7 \%$ ) is as shown in table 6 .

\section{Antibiotic susceptibility pattern of MRSA and MSSA isolates}

The isolates of $S$. aureus were broadly categorized into two groups: MRSA and MSSA. Both the groups of $S$. aureus showed marked variation in the sensitivity pattern against common antibiotics.

It was observed that, for MRSA, the most effective antibiotic was cefotaxime $(n=83$, $81.37 \%)$ followed by gentamicin $(n=81$, $79.41 \%)$ amikacin $(\mathrm{n}=77, \quad 75.49 \%)$, chloramphenicol $(n=76,74.50 \%)$. Similarly,

highest resistance of MRSA was with penicillin $(n=93,91.17 \%)$ followed by ciprofloxacin $(n=85$, $83.3 \%)$ and cotrimoxazole $(n=77,75.49 \%)$ is in table 7 .

\section{Conclusion}

MRSA has acquired resistant to previously effective antimicrobials including in the penicillin, methicillin in comparison over the past 50 years. In hospital infected patients and health carriers are also important source of nosocomial S. aureus [26, 27]. Out of 278 S. aureus isolates isolated from pus samples, 102 (36.69\%) were methicillin-resistant (MRSA). From this study it can be concluded that MRSA infection in pediatric patients is still one of the most threatening infections in hospitals of Nepal. The rate of MRSA occurrence was found to be higher in inpatients compared to outpatients and the male patient than female patients. The highest number of MRSA isolates was found in 1-5 age

Table 7. Antibiotic susceptibility pattern of MRSA and MSSA isolates

\begin{tabular}{lcccccccc}
\hline Antibiotics & \multicolumn{4}{c}{ MSSA(n=176) } & \multicolumn{5}{c}{ MRSA(n=102) } \\
\cline { 2 - 9 } Used & Sensitive & $\%$ & Resistant & $\%$ & Sensitive & $\%$ & Resistant & $\%$ \\
\hline Amikacin & 163 & 92.61 & 13 & 7.38 & 77 & 75.49 & 25 & 24.50 \\
Ciprofloxacin & 91 & 51.70 & 85 & 48.29 & 17 & 16.60 & 85 & 83.33 \\
Cotrimoxazole & 93 & 52.84 & 83 & 47.15 & 25 & 24.5 & 77 & 75.49 \\
Cefoxitin & 176 & 100.0 & 0 & 0.0 & 0 & 0.0 & 102 & 100.0 \\
Erythromycin & 114 & 64.77 & 62 & 35.22 & 59 & 57.84 & 43 & 42.15 \\
Cloxacillin & 151 & 85.79 & 25 & 14.20 & 76 & 74.50 & 26 & 25.49 \\
Cefotaxime & 161 & 91.47 & 15 & 8.52 & 83 & 81.37 & 19 & 18.62 \\
Penicillin & 35 & 19.88 & 141 & 80.11 & 9 & 8.82 & 93 & 91.17 \\
Ofloxacin & 88 & 50.00 & 88 & 50.00 & 26 & 25.49 & 76 & 74.50 \\
Chloramphenicol & 165 & 93.75 & 11 & 6.25 & 76 & 74.50 & 26 & 25.49 \\
Gentamicin & 167 & 94.88 & 9 & 5.11 & 81 & 79.41 & 21 & 20.59 \\
\hline
\end{tabular}


group patients. The most effective antibiotic against MRSA in pediatric patients was found to be gentamicin. Similarly, chloramphenicol, cefotaxime were also found to be effective against MRSA infections whereas penicillin was found to be the least effective antibiotic to treat MRSA infections where $89.92 \%$ of $S$. aureus isolates showed resistance towards this antibiotic.

\section{Acknowledgements}

Authors would like to thank Kanti Children's Hospital (KCH), Maharajgunj, Kathmandu, Nepal for laboratory facilities. Thanks also go to Department of Microbiology and Department of Chemistry, Tri-Chandra Multiple Campus, Tribhuvan University, Kathmandu, Nepal for a technical support.

\section{References}

1. Shittu AO, Jonson L: Antimicrobial susceptibility patterns and characterization of clinical isolates of Staphylococcus aureus. BMC Infect Dis 6. 2006, 125: 2334-2336.

2. Rehm SJ: Staphylococcus aureus: the new adventures of a legendary pathogen. Clev Clin J Med. 2008 75(3): 177-180, 183-186, 190-192.

3. Mallick SK, Basak S: MRSA-too many hurdle to overcome: a study from Central India. Trop Doct. 2010 40(2): 108-110.

4. Foster TJ: Colonization and infection of the human host by staphylococci: adhesion, survival and immune vasion. Vet Dermatol. 2009 20(4-5): 456-470.

5. Otto M: MRSA virulence and spread. Cell Microbiol. 2012, 14(10): 1513-1521.

6. Chambers HF: The changing epidemiology of Staphylococcus aureus. Emerg Infect Dis. 2001 7(2): 178-182.

7. Ray $\mathrm{P}$, Gautam V, Singh R: Methicillin-resistant Staphylococcus aureus (MRSA) in developing and developed countries: implications and solutions. World Health Organization (WHO), Special issue on Antimicrobial Resistance in SouthEast Asia. Regional Health Forum (WHO South-East Asia region) 201115

8. Sanford MD, Widmer AF, Bale MJ, Jones RN, Wenzel RP: Efficient detection and long-term persistence of the carriage of methicillinresistant Staphylococcus aureus. Clin Infect Dis. 1994 19: 1123-1128.

9. Cesur S, Cokca F: Nasal carriage of methicillinresistant Staphylococcus aureus among hospital staff and outpatients. Infect Control Hosp Epidemiol. 2004 25: 169-171.

10. Creechs CB, Kernodle DS, Alsentzer A, Wilson C, Edwards KM: Increasing rates of nasal carriage of methicillin-resistant Staphylococcus aureus in healthy children. Pediatr Infect Dis J. 200524 (7): 617-621.

11. Eveillard M, Martin Y, Hidri N, Boussougant $Y$, Joly GM: Carriage of methicillin-resistant Staphylococcus aureus among hospital employees: prevalence, duration, and transmission to households. Infect Control Hosp Epidemiol. 200425 (2): 114-120.

12. Kenner J, Connor OT, Piantanida N, Fishbain J, Eberly B, Viscount H, et al: Rates of carriage of methicillin-resistant and methicillinsusceptible Staphylococcus aureus in an outpatient population. Infect Control Hosp Epidemiol. 200324 (6): 439-444.

13. Pokharel J, Rawal K: Bacteriological study at T.U teaching Hospital, Kathmandu. Nepal J Institute Med. 1993, 15: 217-221.

14. Lamichhane R, Adhikari RP, Sherchand JB: Study of methicillin-resistance Staphylococcus aureus (MRSA) isolated from different clinical samples. A dissertation submitted to the Central Department of Microbiology, Tribhuvan University, Kirtipur, Nepal 1999.

15. Bhandari RR: Prevalence and antibiotic sensitivity pattern of methicillin-resistance Staphylococcus aureus (MRSA) in Bir Hospital. A dissertation submitted to the Central Department of Microbiology, Tribhuvan University, Kirtipur, Nepal 2002.

16. Kumari N, Mohapatra TM, Singh YI: Prevalence of methicillin-resistant Staphylococcus aureus (MRSA) in a tertiary-care hospital in Eastern Nepal. J Nepal Med Assoc. 200847 (170): 53-56.

17. Sanjana RK, Shah R, Chaudhary N: Prevalence and antimicrobial susceptibility pattern of methicillin-resistant Staphylococcus aureus (MRSA) in CMS- Teaching Hospital: a preliminary report. JCMS-Nepal. 2010, 6: 1-6.

18. Tiwari HK, Das AK, Sapkota D, Sivrajan K, Pahwa VK: Methicillin-resistant Staphylococcus aureus: Prevalence and antibiogram in a tertiary care hospital in western Nepal. J Infect Dev Ctries. 2009 3: 681684.

19. Ansari S, Nepal HP, Gautam R, Rayamajhi N, Shrestha S, Upadhyay G, et al: Threat of drug resistant Staphylococcus aureus to health in Nepal. BMC Infect Dis. 2014 14: 157-162.

20. Pant MR, Shrestha D, Thapa S: Antibiogram of bacterial species causing skin wound infections. NRMJ. 20182 (3): 53-60.

21. Isenberg H: Clinical microbiology procedure handbook Vol I and II. American Society for Microbiology (ASM) press, Washington DC, New York USA, 1992.

22. CLSI: Performance standards for antimicrobial susceptibility testing. 25 th informational supplement, document M100-S25. Wayne, USA 2105, 35 (3).

23. Bergey DH, Buchanan RE, Gibbons NE: Bergey's manual of determinative bacteriology. Williams and Wilkins USA 1974. 
24. Cheesbrough M: Microbiological tests district laboratory practice in tropical countries, Part-II. Low price edition, Cambridge University Press, India 2006, 01-267.

25. Lencastre DH, Oliveira D, Tomasz A: Antibiotic resistant Staphylococcus aureus: a paradigm of adaptive power. Curr Opin Microbiol. 2007 10(5): 428-435.

26. Lelievre H, Lina G, Jones ME: Emergence and spread in French hospitals of methicillinresistant Staphylococcus aureus with increasing susceptibility to gentamicin and other antibiotics. J Clin Microbiol. 1999 37: 3452- 3457.

27. Pant J, Rai SK, Singh A, Lekhak B, Shakya B, Ghimire G: Microbial study of hospital environment and carrier pattern study among staff in Nepal Medical College Teaching Hospital. NMCJ. 2006 8: 194- 199. 